\title{
Statistical Characteristics of the Output Voltage in Compact Marx Generators
}

\author{
A. RaZi Kazemi And K. Niayesh \\ School of Electrical and Computer Engineering, University of Tehran \\ P.O. Box 14395/515, IR-14395 Tehran, Iran
}

\begin{abstract}
Compact Marx generators are widely used as portable pulse generator in different industrial applications, where high power pulses with rise times of order of some tens of nanoseconds are needed. For those applications, closing switches with very short delay and jitter times have to be used. Considering the fact that the breakdown of spark gap is a statistical process, the delay times of the closing switches are statistical voltage-dependent parameters. As a result, the characteristics of the output voltage of the Marx generator, i.e. its amplitude and rise time, are also statistical parameters. In this paper, taking the statistical behaviour of the breakdown process in spark gaps into consideration, the statistical characteristics of the output voltage have been studied in detail.
\end{abstract}

PACS numbers: $52.75 . \mathrm{Kq}, 41.20 . \mathrm{Gz}$

\section{Introduction}

Compact Marx generators have been used for many years as trigger generators for large systems [1]. Increasing importance is being placed on making the power source and high-voltage drives for these systems compact and light weight, reducing the size and weight allows the system to be integrated and designed in to maximum number of operational scenarios [2]. To be able to adopt the design of the Marx generators to those applications, closing switches with very short delay and jitter times have to be used.

In this paper, taking the statistical behaviour of the breakdown process in spark gaps into consideration, the statistical characteristics of the output voltage have been studied in detail. For this purpose, the closing delay time has been assumed to be voltage dependent for a given configuration of the spark gaps and breakdown voltages of the switches are selected as random variables with normal probability distribution functions.

\section{State space description of compact Marx generators}

The equivalent circuit of the Marx generator with three stages is shown in Fig. 1. The closing switches in Fig. 1 are gas spark gaps with time dependent inductances and resistances during switching-on phase. To be able to consider a generalized $n$-stage compact Marx generator, the method of state space is used to calculate its output voltage. The variables of state space are mesh voltages and spark gap inductance currents. Because of different delay times, the switches of different stages are not closed at the same time and therefore depending on the status of the switches, different circuit configurations have to be considered. The compact Marx is studied in three phases. The matrix of coefficients for the state space description of the circuit for a three-stage Marx generator is shown in Eq. (1). In this case, the variables of state space are: $x^{\mathrm{T}}=\left[\begin{array}{lllllll}V_{1} & V_{2} & V_{3} & V_{4} & V_{5} & V_{6} & i_{\text {sw1 }}\end{array}\right]$. With regard to $\dot{x}=A x$, " $A$ " matrix is calculated, parameter $K$ and matrixes $M, P$ are defined as shown in Eq (2). All stray capacitances $C_{\mathrm{e}}, C_{\mathrm{g}}$ and also $C_{\mathrm{s}}, R_{\mathrm{ch}}$ are the same for each stage

$$
\begin{aligned}
& M \dot{x}=P x \rightarrow \dot{x}=M^{-1} P x \Rightarrow A=M^{-1} P, \\
& M=\left[\begin{array}{ccccccc}
K & -C_{\mathrm{s}} & 0 & -C_{\mathrm{g}} & 0 & 0 & 0 \\
-C_{\mathrm{s}} & K & 0 & 0 & 0 & 0 & 0 \\
0 & 0 & K & -C_{\mathrm{s}} & 0 & -C_{\mathrm{g}} & 0 \\
-C_{\mathrm{g}} & 0 & -C & K & 0 & 0 & 0 \\
0 & 0 & 0 & 0 & C_{\mathrm{e}}+C_{\mathrm{c}} & -C_{\mathrm{s}} & 0 \\
0 & 0 & -C_{\mathrm{g}} & 0 & -C_{\mathrm{s}} & K & 0 \\
0 & R_{\mathrm{ch}} & 0 & 0 & 0 & 0 & l_{\mathrm{sw}}
\end{array}\right], \\
& P=\left[\begin{array}{cccccccc}
\frac{-2}{R_{\mathrm{ch}}} & 0 & \frac{1}{R_{\mathrm{ch}}} & 0 & 0 & 0 & 0 & \\
0 & \frac{-1}{R_{\mathrm{ch}}} & 0 & \frac{1}{R_{\mathrm{ch}}} & 0 & 0 & 1 \\
\frac{1}{R_{\mathrm{ch}}} & 0 & \frac{-2}{R_{\mathrm{ch}}} & 0 & \frac{1}{R_{\mathrm{ch}}} & 0 & 0 & \\
0 & \frac{1}{R_{\mathrm{ch}}} & 0 & \frac{-2}{R_{\mathrm{ch}}} & 0 & \frac{1}{R_{\mathrm{ch}}} & 0 & \\
0 & 0 & \frac{1}{R_{\mathrm{ch}}} & 0 & \frac{-1}{R_{\mathrm{ch}}} & 0 & 0 & \\
0 & 0 & 0 & \frac{1}{R_{\mathrm{ch}}} & 0 & -1 & 0 \\
-1 & 1 & 0 & 0 & 0 & 0 & R_{\mathrm{ch}}+r_{\mathrm{sw}}
\end{array}\right],
\end{aligned}
$$

$K=C_{\mathrm{s}}+C_{\mathrm{e}}+C_{\mathrm{g}}$,

where $C_{\mathrm{s}}$ - capacitance for each stage, $C_{\mathrm{e}}$ - stray capacitance to ground, $C_{\mathrm{g}}$ - interstage stray capacitance.

\section{Simulation results}

To take the statistical behaviour of the closing spark gaps into account, the closing delay time has been assumed to be voltage dependent for a given configuration of the spark gaps. The breakdown voltages of the switches are selected as random variables with normal probability distribution functions. For the configuration simulated here, the breakdown voltages are produced 


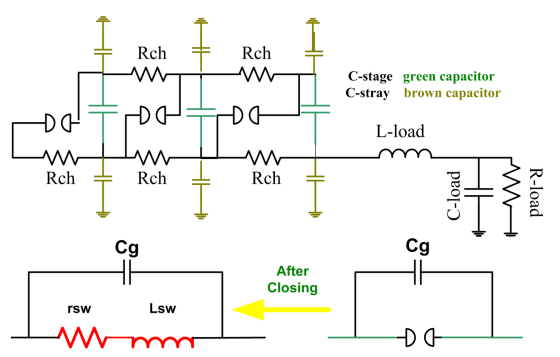

Fig. 1. Circuit diagram of compact Marx generator (left) and model of switch (right).

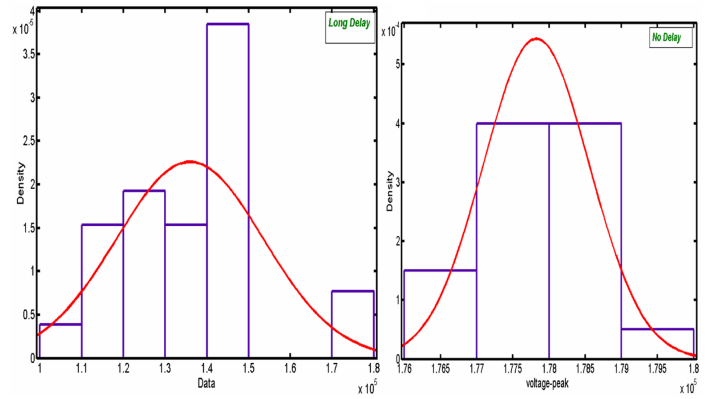

Fig. 2. Normal probability distribution is fitted to amplitude of output voltages (top: long delay, bottom: without delay).

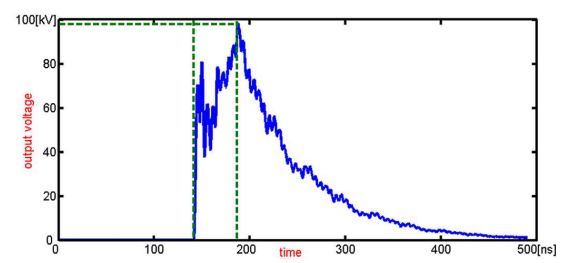

Fig. 3. Output voltage with breakdown voltages $60 \mathrm{kV}$ and closing delay time $70 \mathrm{~ns}$.

TABLE I

Statistical parameter for rise-time (a) and amplitude (b) of the output voltage.

\begin{tabular}{l|c|c}
\hline \hline (a) & Without delay & Long delay \\
\hline mean $[\mathrm{kV}]$ & 177.844 & 135.916 \\
sigma $[\mathrm{kV}]$ & 0.733703 & 17.465 \\
\hline (b) & Without delay & Long delay \\
\hline mean $[\mathrm{ns}]$ & 10.4637 & 22.95 \\
sigma $[\mathrm{ns}]$ & 0.187868 & 16.0775
\end{tabular}

TABLE II

Statistical parameter for producing voltages.

\begin{tabular}{l|c|c|c}
\hline \hline \multicolumn{1}{c|}{ Sigma $[\mathrm{kV}]$} & 3 & 11 & 15 \\
Distribution $V_{\text {out }}$ & & & \\
\hline mean $[\mathrm{kV}]$ & 101.53 & 132.740 & 135.916 \\
sigma $[\mathrm{kV}]$ & 7.13153 & 15.475 & 17.465
\end{tabular}

with mean and standard deviation of $60 \mathrm{kV}$ and $15 \mathrm{kV}$, respectively. To study the influence of the time delays, simulations with two different delay times for the spark gaps have been performed. In one case, the spark gaps are assumed to have no time delay; in other cases the time delays are assumed to be voltage dependent as in [3]. The probability density functions for the amplitude of the output voltage for both cases are shown in Fig. 2. As can be seen in Table, consideration of the time delays results in lower output voltage amplitudes; the rise-time of the output voltage is increased in case of the spark gaps with time delay. Consequently, the closing delay time has severe impact on the rate of rise of the output voltage of the Marx generator. To study the influence of the probability density distribution function of the spark gaps on the amplitude and rise time of the output voltage, different standard deviations for the normal probability density function of the breakdown voltage of the spark gaps have been considered. As can be seen in Table II, the amplitude of output voltage is significantly reduced with decrease of the standard deviation of the probability density function. With breakdown voltage of the spark gaps of $60 \mathrm{kV}$ and the closing delay time of $70 \mathrm{~ns}$, the amplitude of the output voltage is $100 \mathrm{kV}$ (see Fig. 3). To achieve higher output voltage amplitudes, higher breakdown voltages (e.g. $70 \mathrm{kV}$ ) can be selected with accordingly shorter closing delay times (e.g. $60 \mathrm{~ns}$ ) or to use spark gaps with much lower breakdown delay times of 20 ns (for example high pressure spark gaps with reduced gap lengths). In this case, the most probable amplitude of the output voltages of $145 \mathrm{kV}$ can be realized.

\section{Conclusion}

Simulation results indicate that the parameters of the probability distribution function of the breakdown voltage of the spark gaps and the dependence of the breakdown delay times on the breakdown voltage of the gap have considerable influence on the parameters of the output voltage of the Marx generator. For instance, in a three-stage Marx generator with charging voltage of capacitors of about $50 \mathrm{kV}$ used to supply a $60 \Omega$ resistive load, in case of most probable breakdown voltages of $60 \mathrm{kV}$, the most probable amplitude of the output voltage is only $100 \mathrm{kV}$ if the breakdown delay time of the gaps at their most probable breakdown voltage is $70 \mathrm{~ns}$, but using spark gaps with much lower breakdown delay times of 20 ns the most probable amplitude of the output voltage can be increased to $145 \mathrm{kV}$.

\section{References}

[1] R. Mayes, J. Cary, Proc. Power Systems Conference, Coral Springs, 2002, paper no. 02PSC-25, p. 702.

[2] M. Ness, D. Smith, Y. Chau, L. Thomas, R. Cooper, IEEE Trans. Electron Dev. 38, 803 (1991).

[3] S.T. Pai, Qi Zhang, Introduction to High Power Technology, World Sci., Hong Kong 2003, p. 62. 